\title{
EVOLUCIÓN DE LAS TEORÍAS DE LA JUSTICIA CON RESPECTO A LOS DERECHOS HUMANOS YA FORMULADOS Y A LOS FUTUROS DERECHOS
}

\section{María del Rosario Guerra González*}

Resumen: Se analizan teorías de la justicia mostrando sus posibilidades y límites; se completa el pensamiento liberal moderado con concepciones que toman en cuenta las desigualdades culturales y económicas. Se sostiene que si se desarrollara teóricamente el principio de la diferencia, junto con los principios de igualdad y libertad, se tendrían sistemas más justos. Las concepciones teóricas sobre el tema cambiaron en los últimos años, en el presente está en construcción una teoría de la justicia que desea reunir la casi universalidad inserta en el pensamiento de los derechos humanos, con el fomento de la diversidad cultural de los pueblos.

Palabras clave: derechos humanos, diversidad, igualdad, justicia, libertad

Abstract: Theories of justice showing its possibilities and limits are analyzed; moderate liberal thought is completed together with some conceptions which give an important place to cultural and economic inequalities. It is stated that if the principle of difference could be developed with the principles of equality and liberty, systems with more justice could be achieved. The theoretic conceptions about the subject changed in the last years; nowadays a theory of justice is being developed which wants to put together almost all the universality lying in the thought of human rights, emphasizing the cultural diversity of peoples.

Key words: human rights, diversity, equality, justice, liberty.

Doctora en filosofía por la Universidad Iberoamericana. Investigadora del Instituto de Estudios sobre la Universidad de la Universidad Autónoma del Estado de México, profesora de las Facultades de Derecho y Humanidades de la misma universidad. Es miembro del Sistema Nacional de Investigadores de CONACYT, México. Dirección electrónica: rosariogue13@hotmail.com 


\section{Presentación}

Hablar de justicia exige pensar las diferentes situaciones en que viven los hombres y las mujeres del presente, donde reina la diversidad en los ámbitos cultural y económico. Desde el punto de vista cultural se está ante una dualidad: existen factores que universalizan estilos de vida mientras que, simultáneamente, millones de personas viven de manera diferente.

Detrás del proceso que ha creado estilos uniformes hay intereses económicos -en su mayoría no de inversión, sino financieros- que propician una similitud de hábitos que fomenta el consumo, pues se busca que la población compre los mismos bienes. El resultado de esta conducta es una sociedad marcada por las desigualdades económicas. Algunas zonas del planeta globalizado se consideran integrantes de la sociedad de la información y la comunicación, mientras que otras áreas pobladas carecen de energía eléctrica. Las innovaciones técnicas en la industria de las comunicaciones sorprenden cada día. La industria de la guerra está en auge. Mientras algunas personas están pendientes de las innovaciones, otras no tienen lo indispensable para mantenerse vivas. Para mostrar las carencias de muchos basta pensar cuántos niños mueren por deshidratación cada día.

El presente puede verse desde dos ópticas: desde un punto de vista hay un estilo de vida homogéneo y, además, bajo otra visión, existe una multiplicidad cultural viva. Esta tensión entre lo global y lo local es similar al planteamiento de Nagel, en su caso la oposición que es necesario conciliar es entre lo personal y lo interpersonal ${ }^{1}$.

Éste es el problema que se presenta en el presente trabajo: el pensamiento ético-político necesita otro marco conceptual, diferente del clásico, para hacer compatible la filosofía política -principalmente la que se refiere a una teoría de la justicia- con el estilo de vida cotidiano de los pueblos y las personas que constituyen las naciones.

${ }^{1}$ Cfr. Nagel, Thomas, Igualdad y parcialidad, Barcelona, Paidós, 2006. 
Ante el entorno ya descrito se pretende analizar algunas teorías de la justicia expuestas en las tres últimas décadas, mostrar sus límites y completar el pensamiento liberal moderado con concepciones que toman en cuenta las desigualdades culturales y económicas. En este sentido se sostiene la siguiente tesis: si se desarrollara teóricamente el principio de la diferencia, junto con los principios de igualdad y libertad, se tendrían sistemas más justos. Es oportuno aclarar que los bienes y servicios más importantes son valiosos por ampliar la libertad, esto incluye elegir un modo de vida colectivo en el que florezca la persona. El término florecimiento ha sido una sustitución contemporánea de la eudaimonía griega, realizada básicamente por Martha Nussbaum².

El trabajo consta de tres partes: en la primera se analizan diversos modelos teóricos en los que son esenciales la igualdad y la libertad garantizadas para elaborar una teoría de la justicia. El apartado siguiente muestra un paso más: es necesario darle igual rango al principio de la diferencia. En la tercera parte se presenta las carencias de las teorías anteriores. Por último se concluye que, si bien las concepciones teóricas sobre el tema cambiaron sustancialmente en los últimos años, al comienzo del siglo XXI está en construcción una teoría de la justicia que desea reunir la universalidad propia de una ética mínima, inserta en el pensamiento de los derechos humanos, con el fomento de la diversidad cultural de los pueblos. Se finaliza con una serie de ideas presentadas como bosquejo para continuar con futuras investigaciones.

\section{La libertad y la igualdad como conquistas a conservar}

Se suele dividir el pensamiento filosófico-político contemporáneo en dos corrientes: liberales y comunitaristas. Para los primeros, los derechos a salvaguardar son, antes que nada, principios derivados de la condición de persona. Por el contrario los comunitaristas no insisten en la

2 Cfr. Nussbaum, Martha, Terapia del deseo, Barcelona, Paidós, 2003. 
autonomía humana; un ejemplo lo constituye la actitud antikantiana de Charles Taylor ${ }^{3}$.

En este trabajo no se hará un recorrido histórico por las teorías que defienden la libertad y la igualdad, sino que sólo se hablará de la discusión en el presente. Es oportuno adelantar, sin embargo, que asumimos una postura liberal moderada o, dicho con otras palabras, se parte de los derechos de cada persona, de su autonomía moral y desde ahí se construye.

Han existido diferentes concepciones teóricas sobre cómo respetar la autonomía personal: desde imponer esta idea, pasando por tolerar a sociedades no liberales, hasta pensar a la justicia como el "dar y recibir". Encontramos así tres posturas.

Para los primeros, los ideales liberales -entendiendo por tales a los derechos fundamentales- son una conquista de la humanidad que es necesario conservar y que, además, pueden imponerse a los pueblos no liberales. Aquí se ubica al filósofo argentino Ernesto Garzón Valdés, quien ha concebido mecanismos para evitar que un día, dentro de sistemas democráticos, se resuelva derogar las normas que garantizan los derechos básicos. Pero a pesar de aplaudir el lugar que ocupan los derechos de las personas en el pensamiento de este autor, se considera que Garzón Valdés no es capaz de reconocer la validez de enfoques culturales diferentes del europeo, los cuales tienen una concepción de la relación causa-efecto distinta y que no violan principios éticos que reconocen la autonomía de la persona como agente moral. Dentro de su postura es fundamental la definición de bienes básicos. Para Garzón estos bienes son los necesarios para realizar cualquier plan de vida, incluyendo la acción del individuo como agente moral. De ello deriva que, si los miembros de una comunidad no comprenden la importancia de éstos, se justifican medidas paternalistas. El autor dice: "En efecto, la no aceptación de la garantía de los propios bienes básicos es una clara señal de irracionalidad o de ignorancia de relaciones causales elementales. En ambos casos, quien no comprende su relevancia puede ser incluido en la categoría de

${ }^{3}$ Cfr. Taylor, Charles, Las fuentes del yo, Barcelona, Paidós, 1996, p. 28. 
incompetente básico"4. "Incompetentes", “irracionales", adjetivos adjudicados al otro, al que piensa distinto al que emite el juicio.

Una segunda opción liberal consiste en aceptar el estilo de vida de cada pueblo, siempre que allí se respeten los derechos humanos. Ésta es la actitud asumida por John Rawls cuando dice:

Queremos preguntar si el pluralismo razonable dentro o fuera de los pueblos es una condición histórica con la cual debemos reconciliarnos. Si bien podemos imaginar lo que sería un mundo feliz, en el cual todos y cada uno de los pueblos tendrían la misma fe que nosotros, este ejercicio queda excluido por la naturaleza y la cultura de las instituciones libres. Para mostrar que no hay que lamentar el pluralismo razonable, debemos indicar que, dadas las alternativas socialmente factibles, su existencia da paso a una sociedad con más justicia política y con más libertad 5 .

Así plantea su enfoque del derecho de gentes, donde establece las condiciones que debe reunir toda organización política del presente para ser incluida en la comunidad de naciones. El derecho de gentes es un conjunto de conceptos políticos, principios de derecho, justicia y bien común, similares, aunque más generales, a las ideas presentadas en Una teoría de la Justicia. Al autor le interesa aplicar los principios de la justicia, propios de una entidad nacional, a la comunidad internacional. Para hacerlo analiza los "límites de la tolerancia". El debate que abrió este pensador justifica su inclusión en el tema que nos ocupa. No toma como referencia conceptual a la justicia distributiva, propia del interior de un Estado soberano formado por ciudadanos, sino que parte de los mínimos exigibles a los pueblos para garantizar que sus miembros tengan la condición de personas, propia del pensamiento de los derechos humanos.

4 Garzón Valdés, Ernesto, "El problema ético de las minorías étnicas", en León Olivé, Ética y diversidad cultural, México, UNAM-FCE, 2004, p. 46.

${ }^{5}$ Rawls, John, El derecho de gentes, Barcelona, Paidós, 2001, p. 24. 
Rawls entiende que son los miembros del grupo quienes deben resolver cambiar su estilo de vida o conservarlo; solamente debe exigirse el derecho, garantizado, de discrepar con las autoridades de la comunidad que establecen un estilo jerárquico tradicional. Para este autor los miembros de una sociedad jerárquica no son considerados ciudadanos libres e iguales como en las sociedades liberales, pero son los responsables del cambio dentro del grupo social, con lo que existe la posibilidad de escuchar sus voces $\mathrm{y}$, si bien esto no se produce con los mecanismos de las democracias, pueden existir otros métodos propios de esa sociedad. Explícitamente Rawls dice que el gobierno de una sociedad jerárquica tiene la obligación de tomar en serio el disenso y debe responderlo; es decir, no puede rehusarse diciendo que quienes se expresan son incompetentes. Queda garantizada, así, la posibilidad de modificaciones diversas.

El tema anterior no está planteado de manera similar en Garzón Valdés. En el enfoque de éste los representantes de las comunidades indígenas "deben estar dispuestos a abandonar" reglas o principios de comportamiento que aumentan su vulnerabilidad. Es una obligación: deben cambiar; es el deber de dinamización para evitar que las instituciones sean suicidas. Para Garzón Valdés el Estado-nación está por encima de los pueblos.

Las diferentes cosmovisiones son parte de la diversidad cultural y ésta no sólo es un hecho, sino que es fuente de amplias posibilidades. Ningún hombre ni ninguna cultura están en posesión de la verdad absoluta. Cada pueblo encuentra algo; hoy se puede crecer mirando a pueblos diferentes que muestren diversas caras de la realidad, teniendo presente que ninguna faz es superior.

Una tercera postura aparece frente a las respuestas de Rawls: es la propuesta de Paul Ricoeur, quien entiende que si el derecho busca solamente la convivencia pacífica entre hombres que son "lobos del hombre" -tal como lo presentó Hobbes ${ }^{6}$-, justicia significa equilibrio, es

"Nussbaum denomina "egoísta" a esta forma de contractualismo, donde los principios políticos se fundan exclusivamente en el beneficio mutuo, sin premisas 
un valor que permite limitar el poder del más fuerte evitando su arbitrariedad. Si, por el contrario, los hombres son diferentes, si unos han recibido mucho y otros poco, entonces la justicia es la virtud de dar y recibir.

El tema analizado en esta parte del artículo gira alrededor de conservar la libertad y la igualdad justificadas teóricamente desde el siglo XVIII-aunque la práctica diste de lo escrito en documentos. El aporte de los filósofos de fines del siglo XX ha sido encontrar reglas justas en el reparto de cargas y beneficios sociales.

El problema aparece al pretender decir en qué consiste ser justo, porque no hay acuerdo en las respuestas, a pesar de que en el sistema aristotélico cada virtud es un medio entre un exceso y un defecto. Pero el planteamiento no es tan simple. La primera dificultad consiste en averiguar si la justicia rige la distribución de cualquier tipo de bienes en relación con la prosperidad y la adversidad, lo que significa distribuir bienes a compartir y cargas a soportar. Así es el planteamiento de Rawls; para Ricoeur, sin embargo, antes habría que demostrar que realmente toda sociedad, a través de sus instituciones, distribuye ventajas y desventajas. Esto significa que al analizar la idea de justicia hay que partir de la imagen de una sociedad cuyos miembros no sólo quieren vivir juntos, sino que, además, tienen un voto de cooperación y reglas de reparto.

La cuestión radica en puntualizar de qué manera debe hacerse la distribución de partes. La igualdad aritmética no presenta dificultades: todos los hombres tienen igualdad ante la ley, casos iguales son incluidos dentro de la misma norma, etc. Pero la distribución de bienes económicos y sociales no se realiza con igualdad aritmética sino con igualdad proporcional, como lo plantea Rawls ${ }^{7}$. Se trata de la igualdad en

morales. Cfr. Nussbaum, Martha, Fronteras de la justicia, Barcelona, Paidós, 2007, p. 69.

${ }^{7}$ En Rawls los individuos escogen dos principios. El primero asegura el máximo de libertad para cada uno, compatible con la libertad de los otros, aquí aparece el pensador liberal. El segundo principio justifica una desigualdad provechosa para todos, es la afirmación básica que ha merecido múltiples comentarios. Primer principio: Cada persona ha de tener un derecho igual al más extenso sistema total 
una proporción similar a la matemática, igualdad de relaciones (la relación entre la contribución de un individuo y su parte).

Por su parte, Ricoeur retoma la relación de amistad; ésta es, en el trato interpersonal, lo que la justicia pensada como igualdad es en las relaciones institucionales, lo cual permite que exista un otro en cada uno y supone la solicitud. Esta justicia, además, amplía la amistad, porque la extiende a la ciudad, al Estado, a la humanidad.

Nuevamente encontramos otra dualidad: el formalismo imperfecto, teleológico y el formalismo perfecto, deontológico. Este formalismo perfecto deontológico exige la ficción del contrato social. Para el filósofo francés esta hipótesis es inevitable para justificar el reparto; por lo tanto, una ficción o una hipótesis están en la base de la teoría analizada, situación grave, porque el sustento es algo que puede ser desechado. Ricoeur expresa:

Este encuentro entre una perspectiva deliberadamente deontológica en materia moral y la corriente contractualista no tiene nada de contingente. La ficción del contrato tiene por fin y función separar lo justo de lo bueno, sustituyendo en el procedimiento de una deliberación imaginaria todo compromiso previo concerniente a un pretendido bien común. Según esta hipótesis, es al procedimiento contractual al que se le supone que engendra el o los principios de justicia ${ }^{8}$.

El autor entiende que hay una gran diferencia entre la noción de autonomía, fundamento de la moralidad, y la noción de contrato, base de la concepción deontológica. La autonomía y la dignidad van juntas como

de libertades básicas compatible con un sistema similar de libertad para todos. Segındo principio: Las desigualdades económicas y sociales han de ser estructuradas de manera que sean para: a) mayor beneficio de los menos aventajados, de acuerdo con un principio de ahorro justo, $y \mathrm{~b}$ ) unidos a los cargos y las funciones asequibles a todos, en condiciones de justa igualdad de oportunidades. Cfr. Rawls, John, Teoría de la justicia, México, F.C.E., 1997, p. 280.

${ }^{8}$ Ricoeur, Paul, Amor y justicia, Madrid, Caparrós Editores, p. 43. 
principios a respetar en cada acto humano, se pueden insertar en la realidad cotidiana, mientras que la idea de contrato es una ficción, una hipótesis de trabajo.

Frente a todo esto, Ricoeur opta por otro camino donde relaciona los conceptos amor y justicia para evitar que esta última sea solamente un equilibrio instrumental, práctico, que únicamente permita la convivencia pacífica. Para que la justicia sea un valor que guíe la acción necesita ser algo más y el autor encuentra ese 'algo más' en el amor al prójimo, por ello habla del amor y su estatuto normativo en el discurso. La persona que ama tiene obligaciones con el otro, ésta es la base de la justicia como valor: un compromiso con los demás, más que una convivencia pacífica que permita solucionar los conflictos.

La justicia en Ricoeur está a medio camino entre dos extremos, ya vimos cómo necesita nutrirse de amor. Esta estructura dialéctica se da también en otro binomio: lo bueno y lo legal. Estos dos términos también son opuestos, el primero tiene una orientación teleológica y el segundo una concepción deontológica. Es orientación teleológica, porque la justicia es la aspiración de una vida buena, por lo tanto, es un télos, más que un acuerdo entre seres racionales. En esta aspiración está incluida la diferencia al lado de la libertad y la igualdad.

\section{El principio de la diferencia como bien a custodiar dentro de la justicia}

Los planteamientos anteriores conducen a otra alternativa teórica. En la primera posición -ya analizada- los ideales liberales pueden imponerse porque son la única forma de vida correcta; en la segunda postura se toleran otros estilos de vida siempre que respeten los derechos humanos básicos. Ahora se propondrá dar un paso más: no sólo tolerar lo diferente sino apoyar, desde la acción del Estado, la continuidad de las formas de vida de las minorías, exigiendo que éstas respeten los derechos fundamentales, para integrar así una teoría de la justicia.

La igualdad de la existencia humana es amenazada en el mundo moderno y de la misma manera peligra la libre expresión de las diferencias. 
Hablar de sociedad hoy es hablar de igualdad y de diferencias, al referirse a la justicia es indispensable contemplar esta otra dualidad. Después de la Segunda Guerra Mundial, en efecto, los derechos humanos pretendieron proteger a miembros de grupos minoritarios a través de los derechos civiles y políticos básicos, prescindiendo del grupo cultural al que pertenecían. Se pensó que el derecho a la libertad de creencias, la libre expresión y la asociación permitían garantizar el desarrollo cultural sin necesidad de que hubiera un tratamiento especial del tema. La Declaración Universal de Derechos Humanos y los sucesivos Pactos permitieron así establecer principios válidos en numerosos Estados, donde se establecía la autonomía y dignidad de la persona, pues primaba la idea de igualdad. Al comenzar el siglo XXI, la igualdad ya consagrada es importante que sea mantenida, pero es insuficiente; se necesita pensar una teoría de la justicia que incluya las diferencias entre los pueblos de manera permanente, por lo tanto, el Estado tiene la responsabilidad de emprender acciones para conservar la diversidad.

Para argumentar en el sentido arriba indicado se recurrirá al pensamiento del profesor canadiense Will Kymlicka y del mexicano Luis Villoro. El primero se refiere básicamente a la diversidad cultural dentro de las teorías de la justicia que pretendan justificar el pensamiento de los derechos humanos; el segundo parte de la conciencia de exclusión de las minorías.

Kymlicka presenta numerosas dificultades que todavía están por resolverse, para defender los derechos de culturas minoritarias: ¿qué lenguas deben adoptarse en trámites oficiales e inclusos en los parlamentos?, ¿se deben trazar fronteras internas para que las minorías nacionales sean mayoría en determinadas zonas?, ¿deberían distribuirse los poderes políticos con proporcionalidad étnica o cultural?, ¿se deberían proteger las zonas donde vivieron originalmente los indígenas de la colonización, la modernización o la explotación de recursos naturales? y ¿qué grado de integración cultural puede exigirse a inmigrantes y a refugiados antes de otorgarles la ciudadanía?? $\mathrm{El}$ planteamiento de estas

${ }^{9}$ Cfr. Kymlicka, Will, Ciudadania multicultural, Barcelona, Paidós, 1996, p. 18. 
preguntas sugiere pensar las posibles respuestas, lo cual no significa que éstas se respondan afirmativamente; pero es necesario que se analice la realidad bajo otros puntos de vista.

Kymlicka considera que el pensamiento de los derechos humanos no da soluciones a problemas candentes dentro de pueblos multiculturales; aunque se puede partir de ellos. Para complementarlos sostiene que una teoría de la justicia omniabarcadora incluirá tanto derechos universales, asignados a los individuos independientemente de su pertenencia de grupo, como determinados derechos diferenciados de grupo, es decir, un 'estatus especial' para las culturas minoritarias. El mérito del autor no radica en proponer esta complementación, sino en explicar cómo coexisten los derechos de las minorías con los derechos humanos y cómo los primeros están limitados por la libertad individual, la democracia y la justicia social.

Existen grupos étnicos y nacionales que pretenden ignorar las libertades personales, e incluso suprimirlas en la práctica, pero, en otros casos, el respeto por las libertades de las minorías puede ampliar las libertades de los individuos, porque éstas se desarrollan dentro de una cultura. Libertades de las personas significa derechos básicos. Así, es esencial que dentro del liberalismo exista la posibilidad de que cada hombre elija su plan de vida, e incluso que lo vaya modificando a medida que intenta cumplirlo. Detrás de este pensamiento está la postura kantiana, al establecer que la persona no tiene precio sino dignidad. Se trata del principio de la autonomía ${ }^{10}$. Cambiar lo que se ha propuesto vivir exige otra condición, además de poder elegir: conocer otros puntos de vista sobre la vida buena, ser capaz de examinarlos y poder alejarse del grupo donde se ha vivido, si así se desea. Por esto son derechos básicos el acceso a la educación, la libertad de asociación y de expresión. Con estos medios se amplía el horizonte vital. Así, cada persona tiene el derecho de cuestionar las tradiciones de su pueblo. Estos derechos necesitan estar

10 Cfr. Kant, Immanuel, Fundamentación de la metafísica de las costumbres, Madrid, Espasa Calpe, 1994, pp. 103-104. 
garantizados, aunque la mayoría cuestionará detalles y permanecerá dentro de su nación, pues emigrar tiene un costo muy alto.

El planteamiento anterior reúne dos principios: derecho a elegir la propia vida y conocer otros modelos de vida buena, pero ¿qué hacer con los grupos que no tienen estos puntos de partida? Es posible ignorarlos, combatirlos o ayudarlos a volverse liberales en este sentido. Rawls y Kymlicka apoyan la última opción. Los Estados actualmente liberales no lo fueron en el pasado; por lo tanto, los antiliberales actuales pueden no serlo en el futuro si se convencen de que el cambio los beneficia o, por lo menos, de que permite que algunos sectores vivan mejor, generalmente las mujeres, las castas inferiores y las minorías. Incluso en sociedades liberales hay derechos que falta garantizar; se trata de un proceso en el que se marcha, ninguna sociedad lo ha concluido; así lo muestran los informes emanados de las Naciones Unidas o comunicados de organismos no gubernamentales.

A mediados del siglo pasado, los teóricos propusieron que el Estado no interviniera para ayudar a grupos minoritarios, que dejara una evolución natural. Cada estilo de vida puede atraer a nuevos practicantes o, por el contrario, provocar el alejamiento de aquellos que allí nacieron. Proteger prácticas culturales sería innecesario e injusto. Pero, de facto, no existe tal neutralidad, pues la llamada "omisión bienintencionada" no es posible: el Estado privilegia una lengua, un estilo de comunicación, determinadas festividades y símbolos; aquellas prácticas que no son usadas, de hecho están siendo pospuestas.

Ante la propensión inevitable que tiene el Estado, se justifica balancear estos privilegios con resoluciones que protejan las costumbres de las minorías para crear una situación justa. Así, Kymlicka propone se proteja a las culturas con medidas que hagan que la sociedad mayoritaria tenga dificultades en radicarse en las zonas donde viven las minorías, por ejemplo, solicitar abundantes requisitos para otorgar residencia, prestar servicios preferentemente en el idioma de la minoría, otorgar derechos de caza y pesca sólo a los nativos. Establece claramente el principio de igualdad: "La argumentación basada en la igualdad sólo aprueba los derechos especiales para las minorías nacionales si verdaderamente existe 
alguna desventaja relacionada con la pertenencia cultural y si tales derechos sirven realmente para corregir dicha desventaja" 11 . Pero el alcance de los derechos de las minorías variará de acuerdo con las circunstancias particulares.

Otro argumento para conceder privilegios a las culturas minoritarias proviene de reconocer el valor de la diversidad, incluso las mayorías se benefician con la existencia de lo diferente. Como puede observarse, en este razonamiento no se apela a la justicia, como en los casos anteriores, sino al interés. Otros estilos de vida pueden ser adoptados si lo exigen las circunstancias; por ejemplo, el estilo de producción indígena, acorde con el ambiente, puede ser imitado ante la destrucción de las plantaciones industriales. Pero esto se da cuando los miembros de las minorías se incorporan a la cultura dominante. Se ha buscado, a través de medidas proteccionistas, que las personas puedan permanecer dentro de sus grupos originales. Además, es difícil fundamentar medidas gubernamentales que protejan a las culturas minoritarias con base en este argumento, porque para la mayoría el beneficio de tener otros modelos de conducta es ocasional, mientras que el costo es permanente y caro. Kymlicka considera que el precio que pagaría la mayoría sólo es justificable cuando es necesario para "no fomentar las ventajas de los miembros de las mayorías, sino para evitar sacrificios aún mayores a los miembros de la minoría nacional" 12 .

Otra alternativa teórica que incluye la diferencia es la presentada por Villoro, quien afirma dos ideas: en primer lugar, propone tener en cuenta el contexto social formado por hombres y mujeres concretos, es decir, dejar de lado los modelos hipotéticos, y, en segundo término, parte de un disenso crítico.

Según este filósofo el consenso social se ha invocado para mostrar la legitimidad del poder; la discrepancia abre la puerta al disenso. Por esto dice Villoro: “podríamos interpretar la 'exclusión del otro' como una acción pragmática que presentaría dos niveles: 1) la exclusión del otro

\footnotetext{
${ }^{11}$ Kymlicka, op. cit., p. 154.

12 Ibid., p. 172.
} 
respecto al orden del derecho vigente, pero incluido en ese orden, y 2) la exclusión del otro como sujeto de una comunidad de comunicación."13

Dada la argumentación anterior, el punto de partida de Villoro para elaborar una definición de justicia es la conciencia de exclusión del discrepante, el cual proyecta un nuevo orden social donde sea incluido. El discrepante parte de su interés, no lo mueve una racionalidad que reconozca intereses ajenos, ni actúa por un sentido altruista, sino que busca una nueva situación justa para su persona -planteamiento similar al de Rawls en Teoria de la justicia. Con esos motivos, sin embargo, establece un nuevo orden válido para todos los excluidos; por lo tanto, la acción es interesada y a la vez universalizable. Villoro afirma: "podemos detectar una nota propia de todo concepto de justicia, en cualquier circunstancia histórica. Sólo que ésta sería negativa. La justicia que se demanda es, en todo caso, la no exclusión"14. La consecuencia de esta postura es exigir una nueva crítica a la universalidad de los derechos humanos.

Villoro insiste en recordar el contexto histórico de toda formulación. Así, la exclusión de la clase media burguesa lleva a los derechos del hombre en el siglo XVII, el siglo XX ha reclamado la no exclusión de una vida personal con libertad ante los totalitarismos y en la actualidad se necesita un conjunto de derechos básicos que permitieran disfrutar cualquier otro derecho. Entiende que las libertades son derechos básicos, pero en muchas ocasiones no pueden ejercerse, porque se necesita satisfacer necesidades previas: sobrevivencia (alimento, vestido, habitación) y convivencia (pertenencia a una comunidad). Villoro llama "libertades de realización" a la posesión de bienes que permiten elegir un plan de vida y realizarlo.

En consecuencia, las exclusiones a que pueden estar sujetas muchas personas de amplios sectores no son simplemente las de las libertades, sino otras carencias cuya supresión sería para muchos prioritaria. En cada sociedad, en cada sector social, habría que comprobar cuáles son

13 Villoro, Luis, Los retos de la sociedad por venir, México, F.C.E., 2007, p. 27.

${ }^{14}$ Ibid., p. 38. 
los tipos de derechos que reivindican con prioridad. Sólo así podemos llegar a una teoría de los derechos básicos del hombre que fuera aplicable a toda cultura y sociedad ${ }^{15}$.

El autor insiste en la situación cotidiana de las sociedades donde las libertades están incluidas en el orden jurídico y las goza una parte de la población. Otro sector, en cambio, las tiene formalmente, pero en la realidad de cada día vive una situación de dependencia que no le permite ejercer su voluntad y se le exige plegarse a las decisiones ajenas porque de lo contrario perdería el trabajo o, en casos más graves, la vida. Por ello afirma:

La justicia podría expresarse mediante un enunciado negativo: la noexclusión de la pluralidad de culturas, no-exclusión del bien común de la sociedad, no-exclusión en el cumplimiento universal de lo debido. La justicia de no-exclusión que, no por expresarse en un enunciado negativo, es una idea regulativa para el porvenir, abre un horizonte a la justicia y sólo el horizonte hace posible el camino ${ }^{16}$.

En síntesis, para la solución que se propone, el Estado tiene la obligación de intervenir por una razón de justicia, para igualar las condiciones de los grupos mayoritarios y minoritarios, y no puede favorecer a las minorías en cuyo interior no existan las igualdades básicas o derechos fundamentales.

\section{Carencias en las teorías anteriores}

Se ha presentado el pensamiento liberal con diferentes matices: una postura que cree tener todas las respuestas - como es el caso de Garzón Valdez-, una actitud más moderada en el Derecho de gentes de Rawls, un llamado a concebir la sociedad como un lugar donde unos somos

\footnotetext{
15 Ibid., p. 40.

${ }^{16}$ Ibid., p. 113.
} 
deudores de otros, como lo plantea Ricoeur, y, por último, el concepto de un modelo multicultural tal como aparecen en Kymlicka y Villoro.

A pesar de esta evolución se presentarán omisiones alrededor de tres temas: olvidar derechos económicos claves como el derecho al crédito, no limitar derecho de propiedad, no considerar al resto de los seres vivos ni al planeta. No se analizan los temas presentados por Nussbaum como tópicos no tratados: derechos de las personas con discapacidad, de los animales y de los hombres que viven en lugares con serios obstáculos socio-económicos; se considera que ella ya los ha agregado a las teorías de la justicia ${ }^{17}$. Existen otras consideraciones ausentes -además, cada día la humanidad encontrará nuevos aspectos a custodiar- pero por ahora es suficiente con pensar las tres situaciones indicadas.

En primer lugar aparece la situación crediticia. En 1974, a Muhammad Yunus lo asustaban sus elegantes teorías económicas frente al hambre que vivía Bangladesch. Así comenzó a conectarse con la realidad vecina al campus universitario, a aprender de las personas pobres y a responder con la creación de préstamos para autoempleo. Han pasado los años y el banco fundado para permitir el autoempleo tiene sucursales en alrededor de cien países.

Para Yunus pensar en justicia exige tener presente a la pobreza, pero señala cómo los economistas no buscan las causas de la pobreza de un sector específico de la sociedad, sino que estudian la pobreza de los países; piensan que cuando aumente la prosperidad económica como consecuencia habrá menos pobres. Desde este punto de vista lo que se necesita hacer es obtener procesos de desarrollo, no se analiza el hambre que sienten las personas ni la vivienda digna de la que carecen y, para este autor, el problema sigue.

Existe la posibilidad de otro enfoque, es el propuesto por el fundador del banco Grameen. Consiste en asegurar servicios financieros para las personas pobres como parte de la justicia. Originalmente el

${ }^{17}$ Éstos son los temas de su libro Fronteras de la justicia. 
esfuerzo fue centrarse en las mujeres y hombres no propietarios de tierras, quienes no tenían acceso al sistema. Los estudiantes de la universidad elaboraron una lista de personas que dependían de los comerciantes prestamistas. "Allí aparecían los nombres de cuarenta y dos personas que habían pedido prestados, en total, 856 takas, menos de 27 dólares"18. Se necesitaba una respuesta institucional, así surgió el microcrédito. El reporte de Grameen de 2005 indica que se han concedido 199 millones de dólares en préstamos para construir 616 mil casas, con un índice de pago del $98 \%$ en cuotas semanales.

Frente a estas ideas y esta práctica, Yunus considera que el derecho al crédito debe ser incluido como un derecho humano fundamental, junto con los derechos civiles y políticos, porque permite hacer realidad el autoempleo, una vivienda y algún sistema de seguridad social frente a la enfermedad y la vejez, partes básicas a considerar dentro de la justicia.

Otra idea del mismo autor que merece ser incluida en esta reflexión es lo que ha llamado "el poder social del crédito". Los economistas consideran al crédito como un mecanismo que colabora con el comercio y la industria, mientras que para él genera poder económico y de ahí surge poder social. Los bancos excluyen a un sector de la población y con esto dejan fuera a los pobres, muchos etiquetados como "sector informal", sin tener en cuenta que realmente trabajan, en lo que Kart Osner ha llamado "economía popular", el autoempleo.

Yunus define "libre de pobreza" de manera diferente a cómo se lo hace usualmente; no establece un ingreso mínimo que permita comprar bienes indispensables, sino toma en cuenta que las personas tengan los bienes, porque mujeres y hombres de escasos recursos poseen lo básico por otras vías fuera de la compra, por ejemplo, tienen vivienda en una parte del campo de sus progenitores y comen lo que cultivan. En tal sentido, establece diez indicadores: disponer de una casa con tejado de zinc y mosquiteros, camas para cada miembro de la familia, agua potable, ropa abrigada en invierno, letrina, huerto, alimentos todo el año, todos los

${ }^{18}$ Yunus, Muhammad, El banquero de los pobres, Barcelona, Paidós, 2006, p. 53. 
niños van a la escuela y todos los adultos tienen posibilidad de trabajar ${ }^{19}$. Es una enumeración concreta, más práctica que las usuales listas de "bienes básicos" a custodiar dentro de una teoría de la justicia.

El segundo tema ausente en las concepciones de la justicia que tienen en cuenta los derechos humanos se refiere al derecho de propiedad de recursos naturales indispensables para la vida de los hombres y las mujeres. Amartya Sen identifica dos rasgos como característicos de un sistema capitalista: en primer lugar, se basa en los mercados para las transacciones económicas, y, en segundo término, la búsqueda de beneficios y recompensas individuales está fundamentada en la propiedad privada. "Históricamente, el capitalismo no emergió hasta que nuevos sistemas de leyes y prácticas económicas protegieron los derechos de propiedad e hicieron viable una economía basada en la propiedad" 20 .

El derecho de propiedad era considerado por Locke como derecho fundamental, igualado con la vida y la libertad. Los bienes custodiados fueron primero objetos tangibles, como lo son los bienes muebles e inmuebles; luego se reconocieron y protegieron los bienes objeto de propiedad intelectual, ya sea a través de la propiedad industrial invenciones, patentes, marcas, dibujos- o de la custodia de derechos de autor en obras literarias y artísticas, incluyendo los derechos de los productores de fonogramas sobre sus grabaciones.

Con el paso de los años el derecho de propiedad ha rebasado sus límites porque el Estado ha concesionado servicios fundamentales, como el suministro de agua, la atención en centros de salud y la educación. Empresas privadas cumplen estas funciones con controles gubernamentales mínimos, mientras las mujeres y hombres han quedado sin decisión en temas básicos que necesitan ser resueltos por los interesados, como las condiciones de los alimentos que se consumen, el agua que se bebe, las políticas de salud y educación, porque lo que se toma

${ }^{19}$ Cfr., ibid., p. 183.

20 Sen, Amartya, "El capitalismo más allá de la crisis", en Letras libres, Año XI, N 125, mayo 2009, México, p. 16. 
en cuenta son los sistemas de concesiones, reflejo de la propiedad privada.

Ideas similares a las anteriores presenta Shiva"1 en el "Manifiesto para una Democracia de la Tierra":

Las patentes sobre la vida y la retórica de la "sociedad de la propiedad" en la que todo -el agua, la biodiversidad, las células, los genes, los animales, las plantas- es susceptible de convertirse en una posesión expresan una cosmovisión en la que las formas de vida carecen de valor intrínseco, de integridad y de reconocimiento como sujetos. Es una visión de la vida en la que los derechos de los agricultores a sembrar, de los pacientes a disponer de medicinas asequibles y de los productores a gozar de su cuota justa de los recursos de la naturaleza pueden ser vulnerados gratuitamente ${ }^{22}$.

El problema radica en la privatización de la mayoría de los bienes: el conocimiento, el arte, el agua, la salud y la educación. Las dificultades se multiplican cuando estos bienes a custodiar son susceptibles de inversiones en busca de beneficios económicos solamente.

La idea central que se desea destacar es que no todo es susceptible de propiedad y que este aspecto necesita estar incluido dentro de las teorías de la justicia. Sen señala cómo no todo está dentro del mercado. El sistema que permite la concreción de los derechos económicos, sociales y culturales no opera como propiedad privada:

Todos los países ricos del mundo -los europeos, así como Estados Unidos, Canadá, Japón, Singapur, Corea del Sur, Australia y otros- han dependido en parte, desde hace ya bastante tiempo, de transacciones y

21 Doctora en Ciencias Físicas, recibió el Premio Nobel alternativo de la Paz en 1993, directora de la Resarch Foundation for Science, Technology and Natural Resource Policy.

22 Shiva, Vadana, Manifiesto para una democracia de la Tierra, Barcelona, Paidós, 2006, p. 11. 
otros pagos que tienen lugar, en buena medida, fuera de los mercados. Entre ellos están los subsidios de desempleo, las pensiones públicas, diversos rasgos de la seguridad social y las provisiones de educación, salud y otros servicios distribuidos por medio de disposiciones ajenas al mercado. Los derechos económicos relacionados con estos servicios no se basan en la propiedad privada o los derechos de posesión ${ }^{23}$.

Lo que se propone es que se incluya explícitamente ideas similares a las anteriores dentro de las concepciones sobre la justicia.

En tercer término, las concepciones sobre justicia y derechos humanos han olvidado los recientemente reconocidos "derechos de la naturaleza". En esta visión las especies vivientes y la naturaleza no son consideradas "recursos" ni se puede tener sobre ellas derecho de propiedad. De hecho, en algunos lugares la situación está cambiando, esbozo de esto son las últimas constituciones de Bolivia y de Ecuador.

En la misma vertiente se encuentra el pensamiento del filósofo Raimon Panikkar, quien propone que se lleve a cabo un "desarme cultural". Para Pannikar ${ }^{24}$ la cultura occidental es beligerante por una serie de actitudes: competir, buscar lo cuantitativo y mecánico, buscar objetos y posponer al arte, la subjetividad y los sentimientos, entre otras. Esta cultura occidental moderna sobrevalora el progreso, la tecnología, la ciencia, la democracia, el mercado económico mundial, manejando estos conceptos y también manipulando con ellos para obtener objetivos que son acciones, en muchos casos, bélicas.

Todavía estas ideas no son suficientemente escuchadas. En la sociedad occidental, dice Alberto Acosta, "no se considera a la naturaleza como un todo, sino que se reconocen sus elementos en tanto tienen una utilidad inmediata, como la madera, los bananos o los minerales del subsuelo. Allí están los recursos naturales para ser explotados, comprados

${ }^{23}$ Sen, "El capitalismo más allá de la crisis", p. 15.

${ }^{24}$ Cfr. Panikkar, Raimon, La experiencia filosófica de la India, Madrid, Trotta, 1997. 
y vendidos. Hace no mucho tiempo primaba una visión similar en relación a los esclavos" 25 .

El proyecto "Democracia de la tierra", estructurado por Shiva, protege los derechos humanos fundamentales incluyendo el derecho a la vida, al agua, a la comida, a la salud, a la educación, a un puesto de trabajo y un medio de vida, junto con el reconocimiento del respeto a la vida de todas las especies y del mismo planeta. Uno de sus principios establece:

En la Democracia de la Tierra los sistemas económicos protegen los ecosistemas y su integridad; protegen los medios de vida de las personas y satisfacen sus necesidades básicas. En la economía de la Tierra no hay personas, ni especies, ni culturas prescindibles. La economía de la Tierra es una economía viva. Está basada en sistemas sostenibles, diversos y pluralistas que protegen la naturaleza y las personas, que son elegidos por éstas y que obran por el bien común ${ }^{26}$.

Para lograr lo anterior es obvio que la atención está puesta en las economías locales. Aquí aparece el término localización: en tal sentido, sólo se admite la comercialización de productos traídos desde lugares lejanos si no pueden producirse en el lugar.

En oposición a las ideas anteriores puede argumentarse que los gobiernos no pueden ocuparse de tanto; a lo que Sen respondería enfáticamente. En efecto, en 2009, ante la crisis financiera, Sen continúa recordando a Smith y al interés propio de los panaderos y cerveceros dentro del mercado, caso tratado por Rawls:

El fracaso más inmediato del mecanismo del mercado reside en las cosas que el mercado deja de bacer. El análisis económico de Smith fue mucho más allá de dejarlo todo a la mano invisible del mercado. No sólo era un defensor del papel del Estado en la prestación de servicios

25 Acosta, Alberto, Derechos de la Naturaleza. El futuro es abora, Quito, Abya-Yala, 2009 , p. 18.

${ }^{26}$ Shiva, Op. cit., p. 18. 
públicos, como la educación y el alivio de la pobreza (además de exigir una mayor libertad para los indigentes de las que les daban las Leyes de Pobres de su época), también estaba muy preocupado por la desigualdad y la pobreza que podía sobrevivir en una economía de mercado por demás exitosa ${ }^{27}$.

Sen considera que se ha mutilado el pensamiento de Smith, porque éste apoyaba a los mercados y al uso del capital, pero incluía a otras instituciones como los servicios públicos de educación y la presencia de valores, principalmente la prudencia, la justicia, la generosidad y el espíritu público. "Smith llamaba a los promotores de riesgos excesivos en busca de beneficios 'despilfarradores y especuladores', lo que es una buena descripción de los emisores de las hipotecas subprime de los últimos años"28. Se está ante otros pensadores que piensan aspectos no considerados en las teorías políticas. Así, la reflexión de Sen continuó, y en La idea de la justicia ${ }^{29}$ manifiesta que busca conceptos que incluyan maneras de juzgar cómo se reduce la injusticia y a su vez reclama una "argumentación razonada" en lugar de "tú tienes razón en tu comunidad y yo tengo razón en la mía”. Al autor le preocupa la viabilidad de los derechos humanos, que no sean teoría muerta, por esto piensa en un escrutinio sobre el tema. Afirma: "Esto implica la invocación de un proceso interactivo de escrutinio crítico con imparcialidad abierta (incluida la apertura a la información procedente inter alia de otras sociedades y a los argumentos procedentes de cerca y de lejos) lo cual permite debates sobre el alcance y contenido de los presuntos derechos humanos"30. La postura de Sen coincide con lo propuesto en este texto.

${ }^{27}$ Sen, "El capitalismo más allá de la crisis", p. 16.

28 Ibid., p. 17.

${ }^{29}$ Sen, Amartya, La idea de la justicia, México, Taurus, 2010.

30 Ibid., p. 417. 


\section{Reflexión final}

Las desigualdades se incrementan cada día; se dice que el mundo actual es la sociedad de la información, pero millones de personas ni siquiera tienen acceso a la energía eléctrica. En algunos lugares la educación usa las llamadas TICs (tecnologías de la información y la comunicación) mientras niñas y niños no pueden llegar a una escuela primaria porque están trabajando. Ante esta situación las teorías de la justicia necesitan incluir los grados crecientes de desigualdad. Ése fue en parte el intento de Rawls con su Teoria de la justicia, pero la situación ha rebasado el célebre segundo principio planteado por el filósofo norteamericano; no es suficiente con que las desigualdades sean mantenidas en beneficio de los menos aventajados tal cómo él lo manifiesta. Hay un notorio avance al pasar del análisis de la justicia en una "sociedad bien ordenada", propia de la sociedad norteamericana, a pensar en "pueblos" que no tienen obligatoriamente que seguir el modelo de "estados- nación" 31 . El proceso intelectual anterior es positivo pero no es suficiente, porque las peculiaridades culturales de cada pueblo -en los casos que no violan los derechos humanos fundamentales- no son protegidas, solamente son toleradas. Kymlicka da este paso, pero no toma en cuenta de manera enfática los derechos económicos, pospuestos cada día con el pretexto de ser objeto de justicia progresiva. Especial aporte conceptual agregan Sen y Nussbaum, quienes hablan de "capacidades", porque son abiertas y se formularían con un contenido específico en cada época.

Lo que se ha querido mostrar en este texto es la necesidad de continuar con la reflexión en torno a una teoría de la justicia donde se fomente a diferentes estilos de vida -siempre que respeten las libertades fundamentales- y que, simultáneamente beneficie a los hombres y mujeres que son perjudicados dentro del desigual sistema económico, social y cultural actual. La investigación necesita continuar, con el objeto de presentar una ruta. En ese sentido se enumeran, a continuación, bienes

31 Esta evolución se encuentra en el enfoque que aparece en Teoria de la justicia (p. 18) y el posterior planteamiento en Derecho de gentes. 
a proteger dentro de una teoría de la justicia: a) la vida en todas sus formas: vegetales, animales, personas, dentro de un ambiente propicio, (incluye privilegiar lo vivo aún en oposición a intereses financieros); b) la diversidad: razas, lenguas, religiones, costumbres; c) los derechos humanos ya reconocidos: "civiles y políticos", "económicos, sociales y culturales", los de "interés difuso" como el derecho a la paz o al desarrollo; d) el derecho al trabajo y a un salario justo, donde las aptitudes personales pudieran ser desarrolladas (significa proteger el trabajo artesanal y la creatividad en todas sus formas, no es suficiente con los derechos ya garantizados); e) el derecho al crédito; f) el derecho a sobrevivir en épocas de emergencia (incluye contemplar el desempleo o catástrofes naturales).

Una teoría de la justicia necesita especificar los actores responsables de llevarla a la práctica; en primer lugar, el Estado, que tiene su papel fundamental, pero no es el único, la sociedad civil organizada tiene su parte y además, cada hombre y mujer tiene su labor a cumplir.

Recibido: 3/2011; aceptado: 4/2011. 